\section{REAL-LIFE DATA ON GYNECOLOGICAL SARCOMA - THERAPEUTIC STRATEGIES FOR PRIMARY TREATMENT - RESULTS OF THE GERMAN SARCOMA REGISTRY REGSA (NOGGO RU1)}

${ }^{1} \mathrm{E}$ Roser* ${ }^{1},{ }^{1} \mathrm{~F}$ Schmidt, ${ }^{2} \mathrm{D}$ Zocholl, ${ }^{3} \mathrm{~L}$ Hanker, ${ }^{4} \mathrm{ML}$ Poelcher, ${ }^{5} \mathrm{M}$ Frank, ${ }^{6} \mathrm{D}$ Hornung, ${ }^{7} \mathrm{~T}$ Fehm, ${ }^{8} \mathrm{C}$ Traut, ${ }^{9} \mathrm{D}$ Denschlag, ${ }^{10} \mathrm{~B}$ Schmalfeldt, ${ }^{11} \mathrm{~J}$ Terpe, ${ }^{12} \mathrm{~F}$ Marmé, ${ }^{13} \mathrm{G}$ Bauerschmitz, ${ }^{14} \mathrm{M}$ Keller, ${ }^{1} \mathrm{~K}$ Pietzner, ${ }^{1} \mathrm{~J}$ Sehouli. ${ }^{1}$ Charité - Universitätsmedizin Berlin, Charité Campus Virchow Clinic, Department of Gynecology with Center for Oncological Surgery, Berlin, Germany; ${ }^{2}$ Charité - Universitätsmedizin Berlin, corporate member of Freie Universität Berlin and Humboldt-Universität zu Berlin, Institute of Biometry and Clinical Epidemiology, Berlin, Germany; ${ }^{3}$ University Clinic of Schleswig-Holstein, Campus Lübeck, Department of Gynecology and Obstetrics, Lübeck, Germany; ${ }^{4}$ Rotkreuzklinikum Munich, Department of Gynecology, Munich, Germany; ${ }^{5}$ Ortenau Klinikum Offenburg-Kehl, Department of Gynecology and Obstetrics, Offenburg, Germany; 'Diakonissenkrankenhaus Karlsruhe, Vidiakliniken, Department of Obstetrics and Gynecology, Karlsruhe, Germany; 'University Clinic of Duesseldorf, Department of Gynecology, Duesseldorf, Germany; ${ }^{8}$ St. Elisabeth Hospital Köln-Hohelind, Department of Gynecology and Obstetrics, Köln, Germany; ${ }^{9}$ Hochtaunus-Kliniken, Department of Gynecology, Bad Homburg, Germany; ${ }^{10}$ University Medical Center Hamburg Eppendorf, Department of Gynecology, Hamburg, Germany; ${ }^{11}$ Klinikum Südstadt Rostock, Department of Gynecology, Rostock, Germany; ${ }^{12}$ Medical Faculty Mannheim - University Hospital Mannheim - Heidelberg University, Department of Gynecology, Mannheim, Germany; ${ }^{13}$ University Medical Center Göttingen, Department of Gynecology , Göttingen, Germany; ${ }^{14}$ North-Eastern German Society of Gynaecological Oncology, NOGGO e. V., Berlin, Germany

\subsection{6/ijgc-2021-ESG0.258}

Introduction/Background Gynecological Sarcomas (GS) account for only about 3\% of all gynecological malignancies. They are known for their poor prognosis and lack of promising treatment options. Due to the rarity and heterogeneity of GS there is only little consensus regarding the optimal therapeutic strategies throughout all possible situations of the disease. REGSA is by date the largest gynecological registry for sarcoma in Germany.

Methodology Primary inclusion criteria was histologically confirmed diagnosis of sarcoma or STUMP of the female genital tract or sarcoma of the breast. Participating centers were authorized to enter data of sarcoma patients into electronic Case Report Forms. For the work presented here data on therapeutic strategies for primary treatment were analyzed descriptively.

Results From August 2015 till February 2021, 723 sarcoma patients were included by a total of 120 centers. Real-life data on therapeutic strategies for primary treatment was available in 600 cases. 571 patients underwent surgical treatment. In 465 patients a hysterectomy was performed, 200 had no further surgical interventions. An additional salpingoophorectomy was performed in 251 cases. Lymphonodectomy, omentectomy or intestinal resection was performed in less than $15 \%$ each. $21.4 \%$ of patients received chemo- or targeted therapies. Mono chemotherapy was administered more often than a combination chemotherapy. Anthracyclines were the most commonly used substances. 42 patients, mainly patients with Low-grade Endometrial Stromal Sarcoma received an anti-hormonal treatment and 31 patients underwent radiotherapy.

Conclusion Despite the limitations which arise from the structure of a clinical registry, the presented real life data of 600 patients are by date one of the largest analyses of the therapeutic strategies used for GS. Further trials are urgently needed to gain more information about treatment modalities, therapy response and patient-reported outcomes in order to implement new treatment strategies. OLD

${ }^{1 ; 2} \mathrm{D}$ Zouzoulas*, ${ }^{1} \mathrm{D}$ Tsolakidis, ${ }^{3} \mathrm{G}$ Gitas, ${ }^{4} \mathrm{M}$ Zafrakas, ${ }^{1} \mathrm{D}$ Goulis, ${ }^{5} \mathrm{G}$ Douganiotis, ${ }^{2} \mathrm{G}$ Sympilidis, ${ }^{1} \mathrm{G}$ Grimbizis. 'Aristotle University of Thessaloniki, 1st Department of Obstetrics and Gynecology, Thessaloniki, Greece; '2'Theagenio' Cancer Hospital of Thessaloniki, Surgical-Oncologic Breast Cancer Department, Thessaloniki, Greece; ${ }^{3}$ University Hospital Schleswig Holstein, Campus Luebeck, Department of Gynaecology and Obstetrics, Germany; ${ }^{4}$ International Hellenic University - Sindos Campus, Sindos, Greece; ${ }^{5} T$ Theagenio' Cancer Hospital of Thessaloniki, 3rd Department of Clinical Oncology, Thessaloniki, Greece

\subsection{6/ijgc-2021-ESGO.259}

Introduction/Background* Women $\leq 35$ years old with breast cancer constitute a special group. Considering the impact of the disease and its prognosis, these patients face some specific problems, that are not present in older women. What are the prognostic features of the survival rate in very young women with breast cancer?

Methodology Retrospective analysis of very young women with breast cancer from the Surgical-Oncologic Breast Cancer Department at 'Theagenio' Anticancer Hospital, 2003 - 2016. Patient \& tumor characteristics, treatment options and followup information were collected. Univariate - multivariate analyses were conducted and survival rates were calculated.

Result(s)* 129 patients met the inclusion criteria. The median age was 34 years old. 53 patients (41\%) had T1, $36(28 \%)$ had T2, $7(5.4 \%)$ had T3 and $33(25.6 \%)$ had T4 stage tumors. Most women, 114 (88.4\%), had ductal carcinoma in their histology. Furthermore, positive axillary lymph nodes were present in 62 women $(48 \%)$. In the immunochemistry report, 91 patients $(70.5 \%)$ were hormone receptor positive, HER2 was overexpressed in 32 patients (24.8\%) and 27 patients presented with Triple negative subtype. Out of 65 patients tested for Ki-67, 51 (78.5\%), had a high expression (cut off value of 20\%). After adjusting for all possible factors, the risk of recurrence and death was six times higher in the positive lymph nodes group, $(\mathrm{p}<0.001)$. The median diseasefree and overall survival was 133 and $>173$ months, respectively.

Conclusion* Breast cancer in very young women appears with large size and high-grade tumors, high incidence of infiltrated axillary lymph nodes, high Ki-67 expression and intrinsic subtypes with poor prognosis. As a result, these women need to be treated by a multidisciplinary team.

\section{HYPERSPECTRAL IMAGING FOR TISSUE CLASSIFICATION AFTER OVARIAN CANCER SURGERY}

${ }^{1} \mathrm{~S}$ Pérez ${ }^{*},{ }^{2} \mathrm{~N}$ Van de Berg, ${ }^{3} \mathrm{~F}$ Manni, ${ }^{4} \mathrm{M}$ Lai, ${ }^{5} \mathrm{~L}$ Rijstenberg, ${ }^{6} \mathrm{~B}$ Hendriks, ${ }^{7} \mathrm{~J}$ Dankelman ${ }^{5} \mathrm{PC}$ Ewing-Graham, ${ }^{2} \mathrm{G}$ Nieuwenhuyzen-de Boer, ${ }^{2} \mathrm{HJ}$ Van Beekhuizen. ${ }^{1}$ Erasmus $M C$, Radiation Oncology, Rotterdam, Netherlands; '2Erasmus MC, Gynaecologic Oncology, Rotterdam, Netherlands; ${ }^{3}$ Eindhoven University of Technology, Electrical Engineering, Eindhoven, Netherlands; ${ }^{4}$ Eindhoven University of Technology, Biomedical Engineering, Eindhoven, Netherlands; ${ }^{5}$ Erasmus MC, Pathology, Rotterdam, Netherlands; ${ }^{6}$ Delft University of Technology, Biomechanical Engineering, Delft, Netherlands; ${ }^{7} T U$ Delft, Biomechanical Engineering, Delft, Netherlands

\subsection{6/ijgc-2021-ESGO.260}

Introduction/Background* The most important prognostic factor for the survival of advanced-stage ovarian cancer is the completeness of cytoreductive surgery (CRS). Therefore, an 
intraoperative technique to detect microscopic tumours would be of great value. The aim of this pilot study is to assess feasibility of near infrared (NIR) hyperspectral imaging (HSI) for the detection of malignant ovarian cancer, using ex vivo tissue samples collected during CRS.

Methodology In this pilot-study, patients with proven or suspected ovarian cancer planned for CRS were enrolled in the study. Hyperspectral images with 25 spectral bands were acquired from the resected tissues in the wavelength range of 665-975 nm. All hyperspectral data were processed by image calibration and min-max normalisation, glare removal, feature selection and linear support vector machine (SVM) classifier training. The performance of the classification was evaluated by leave-one-out cross-validation.

Result(s)* Ten patients who underwent cytoreductive surgery for advanced-stage epithelial ovarian cancer (EOC) were included in the study, from which 26 tissue samples were imaged, with a total of 26.446 data points that were matched to histopathology. Samples included tissue of the ovaries, fallopian tubes, uterus, omentum and/or part of the intestines. Overall, HSI combined with the SVM classifier was capable to discriminate tumour tissue from non-tumour tissue with a sensitivity of 0.81 , specificity of 0.75 , area under the curve of 0.83 , and Matthew's correlation coefficient of 0.41 .

Conclusion* This pilot study shows that hyperspectral imaging is a promising technique to discriminate ovarian carcinomas from the surrounding tissue. Hyperspectral imaging can scan a whole area, is fast, non-contact, non-invasive and can be used inside the operation room.

\section{ASSESSMENT OF HPV INFECTION AND P16INK4A AND KI67 EXPRESSIONS IN VAGINAL INTRAEPITHELIAL NEOPLASIA}

${ }^{1} Y$ Minareci, ${ }^{2} \mathrm{~N}$ Ak, ${ }^{3} \mathrm{OA}$ Tosun, ${ }^{4} \mathrm{H}$ Sozen, ${ }^{5} \mathrm{~S}$ Önder, ${ }^{4} \mathrm{~S}$ Topuz, ${ }^{4} \mathrm{MY}$ Salihoglu. ${ }^{1}$ Eskişehir Şehir Hastanesi, Gynecologic oncology, Eskişehir, Turkey; ${ }^{2}$ Yozgat Şehir Hastanesi, Yozgat, Turkey; ${ }^{3}$ Medeniyet Universitesi Goztepe Hospital, Gynecologic oncology, Istanbul, Turkey; ${ }^{4}$ Istanbul University Faculty of Medicine, Gynecologic oncology, Istanbul, Turkey; ${ }^{5}$ stanbul University Faculty of Medicine, Pathology, Istanbul, Turkey

\subsection{6/ijgc-2021-ESG0.261}

Introduction/Background* The term vaginal intraepithelial neoplasia (VaIN) refers to a premalignant lesion that has the potential to progress to invasive cancer. VaIN is a very rare disease and represents less than $1 \%$ of all female genital intraepithelial neoplastic lesions. Several studies have shown that high-risk (hr) HPV infection is an important factor in the development of VaIN. Although $\mathrm{p} 16^{\mathrm{INK} 4 \mathrm{~A}} / \mathrm{Ki} 67$ markers have been studied many times in cervical dysplastic lesions, they have rarely been used in vaginal dysplastic lesions. In the present study, we aimed to analyze the role of p16 and Ki67 levels on the progression or regression of VaIN together with HPV infection in vaginal epithelial cells with neoplastic changes.

Methodology Cases of VaIN1, and VaIN2/3 were retrospectively identified from the surgical pathology files at the Department of Pathology, Istanbul Medical Faculty, Istanbul University from 2003 to 2020. A total of 10 cases of VAIN1, and 38 cases of VAIN2/3were identified. The primary endpoints of the study were the recurrence of VAIN and progression to vaginal carcinoma.

Result(s)* Most of HPV positive cases (22 out of 36) were infected with HPV16 subtype (61.1\%). One patient had
HPV18 subtype (5.9\%) infection. There was a significant correlation between the expression of p16INK4A and Ki67 together with the disease recurrence. Patients with strong expression of both $\mathrm{p} 16^{\mathrm{INK} 4 \mathrm{~A}}$ and Ki67 had a significantly higher disease recurrence $(p=0.010)$. Furthermore, we observed that the patients with strong expression of $\mathrm{p} 16^{\mathrm{INK} 4 \mathrm{~A}}$ and Ki67 together, had the recurrence of the disease less than 12 months $(p=0.041)$. Patients with strong expression of both $\mathrm{p} 16^{\mathrm{INK} 4 \mathrm{~A}}$ and Ki67 had a significantly higher disease progression to invasive cancer $(\mathrm{p}=0.015)$. hr HPV positivity was significantly related with moderate or strong expression of $\mathrm{p} 16$ and ki67 $(\mathrm{p}=0.002)$.

Conclusion* Strong expressions of $\mathrm{p} 16^{\mathrm{INK} 4 \mathrm{~A}}$ and Ki67 together in vaginal tissue, had a significantly higher risk of recurrence. Furthermore, with strong expression of both markers together, the disease recurred more rapidly. In addition, strong expression of both markers might be associated with malignant progression. Finally, rate of infection with HPV18 subtype might be less than expected in patients with VaIN. These results need to be confirmed in future prospective studies.

\section{GYNECOLOGIC CANCER SURGERY DURING COVID-19 PANDEMIC: IS IT SAFE ?}

${ }^{1} \mathrm{~F}$ Atalay, ${ }^{1} \mathrm{Z}$ Ozturk Basarrı*, ${ }^{2} \mathrm{Z}$ Uzundurukan. ${ }^{1}$ University of Health Science Dr.AY Ankara Oncology Training and Research Hospital, Gynecological Oncology, Ankara, Turkey; ${ }^{2}$ University of Health Science Dr.AY Ankara Oncology Training and Research Hospital, Statistics, Ankara, Turkey

10.1136/ijgc-2021-ESGO.262

Introduction/Background*:After WHO declared Covid-19 as a pandemic, many scientific organizations, including gynecological oncology and gynecological surgery associations, made new recommendations for treatment protocols for certain diseases. Gynecological cancer surgery could not be performed in many hospitals and medical centers, as they could not provide optiamal conditions for their patients and staff. In this process, our hospital, which was a comprehensive cancer center, was defined as Covid-19 free hospital and established as a reference for other institutions regarding priority oncological surgeries by the regional healthcare authorities.

Methodology The data of the patients who were operated with the diagnosis of gynecological cancer between March 1, 2020 and March 1, 2021 were scanned through files. Demographics, comorbidities, surgical type, complications and COVID-19 status were reviewed. Statistical analyzes were performed using the SPSS 22.0 (Statistical Program Social Sciences) package program. The study was approved by the ethical committee the Institutional Review Board of Dr. AY Ankara Oncology Training and Research Hospital and the Ministry of Health Scientific Research Platform.

Result(s)* The study included a total of 74 patients. Mean age was 58 years (range 16-85). Patients were referred with endometrial $(32 / 74,43,2 \%)$, ovarian $(36 / 74,48,6 \%)$, cervical $(5 / 74$, $6,8 \%)$, or vulvar cancer $(1 / 74,1,4)$. All of the patients underwent open-route laparotomy. Mean hospital stay was 12 days (range 6-51). 13 patients had a postoperative complication (13/74, 17,6\%). 8 patients tested positive for COVID-19 following a Polymerase Chain Reaction(PCR) test, in the postoperative period, after discharge. PCR test for detection of severe acute respiratory syndrome coronavirus 2 (SARS-COV2) was conducted in $73 \%$. The Covid-19 PCR test has been 